\title{
One promise fulfilled, much still to be done
}

\author{
Fiona Godlee editor in chief, The BMJ
}

This year, 2015, was the deadline for some pretty big promises. When these were made it must have seemed a long way off. In an article in the Lancet in 2004 I and others set 2015 as the date when there would be, we hoped, "health information for all" (Lancet 2004;364:295-300). More prominently, 2015 was the deadline for the United Nations' millennium development goals. Now, with much achieved but of course still more to do, we are into the post-2015 development agenda.

But one important promise for 2015 has been fulfilled. The European Medicines Agency said that it would make publicly available the raw data from clinical trials of all newly approved drugs. And despite legal action from the drug industry (doi:10. 1136/bmj.f1636) the agency has pushed ahead, and the new policy is in place. It will be a little while longer-until mid-2016 - before it takes full effect. And the agency can still make restrictions and redactions to protect commercial confidentiality.

However, the fact remains that within two years the public and researchers will be able to read, in full, clinical study reports for all newly approved drugs, whether the trials were conducted by the industry or academia. This is an enormous achievement and something to celebrate. The $B M J$ is doing so by profiling some of the key people who pushed for change and the influential people who listened (doi:10.1136/bmj.g7717).

There is still, of course, much to be done before we can really trust the evidence base for clinical decisions. As the AllTrials campaign (alltrials.net) continues to emphasise, drugs in current use will not be affected by the European Medicines Agency's new policy. So although the future of data transparency may prove better than in the past, we have a huge legacy of secrecy to unpick, compounded by poor and commercially conflicted science. The oseltamivir (Tamiflu) saga has taught us a lot about hidden data (thebmj.com/tamiflu). In particular, it has brought the term "clinical study report" into common parlance. Before the work of the Cochrane review group on Tamiflu, these crucial documents were known only to industry and the regulators.

The statins saga looks likely to yield similarly interesting insights as The $B M J$ and others continue to try to bring the clinical trial data for these most commonly prescribed drugs into the public domain for independent scrutiny (thebmj.com/ open-data). And a recent article in The BMJ reminds us that drugs are not the only aspect of clinical care for which the evidence base is inadequate. Stephen Chapman and colleagues report that a fifth of surgical trials are stopped early and that a third remain unpublished nearly five years after completion (doi:10.1136/bmj.g6870). As summarised in the linked editorial by John Ioannidis (doi:10.1136/bmj.g7089), too many surgical trials are "unregistered, unfinished, unpublished, unreachable, or simply irrelevant."

Ioannidis gives his prescription for what clinical trials should be: well designed, preregistered, asking questions that matter to patients and the public, informed by systematic review of the evidence, well powered and using the best comparators, designed and conducted by triallists without conflicts of interest, and with the raw data publicly available.

I cannot put it better than he does. He writes, "Eventually, randomised controlled trials could be the pride of clinical investigators who collaborate in research that matters, and the best source of information on how to improve health. This is what trials were supposed to be, even if we have almost totally forgotten this over the years."

\section{Cite this as: $B M J$ 2015;350:g7811}

๑ BMJ Publishing Group Ltd 2014 